\title{
Experimental investigation of flow field and string cavitation inside a transparent real-size GDI nozzle
}

\author{
Dmitrii Mamaikin $^{1}$ (1) $\cdot$ Tobias Knorsch $^{2} \cdot$ Philipp Rogler $^{2} \cdot$ Michael Wensing $^{1}$
}

Received: 15 November 2019 / Revised: 6 May 2020 / Accepted: 19 May 2020 / Published online: 17 June 2020

(c) The Author(s) 2020

\begin{abstract}
Gasoline direct injection engines are highly dependent on the spray performance and associated combustion quality. The spray formation depends on many factors, namely internal flow characteristics, injection conditions, ambient conditions and fuel properties. There have been many studies performed to obtain a better understanding of these factors in recent years. In contrast to the others, studies on the internal flow characteristics are often performed numerically, as such, relevant experimental data is still lacking. Experimental investigations of the internal flow, such as flow turbulence, velocity distribution and cavitation are especially challenging under realistic conditions. Therefore, these conditions are generally simplified to diminish the demand for specialized experimental equipment and facilitate the measurements. In this regard, experimental data under relevant conditions are of high interest in the spray community. This work is focused on the internal flow study of multi-hole transparent nozzles under transient conditions at 1:1 geometrical scale. Injection pressure up to 100 bar is applied. The formation and development of string cavitation inside the nozzle hole are observed and presented in detail. For that, a novel ultra high-speed imaging technique at $5 \mathrm{MHz}$ is applied. This technique in combination with the micro particle image velocimetry method, is then able to help to produce the velocity distribution of the internal flow. The velocity data is used further to reconstruct the pressure inside the nozzle by applying the Reynolds-averaged Navier-Stokes equations. Thus, the unique experimental data for the pressure distribution of the liquid fuel is obtained.
\end{abstract}

\section{Graphic abstract}

\section{Experimental investigations are performed inside true scale GDI nozzle}

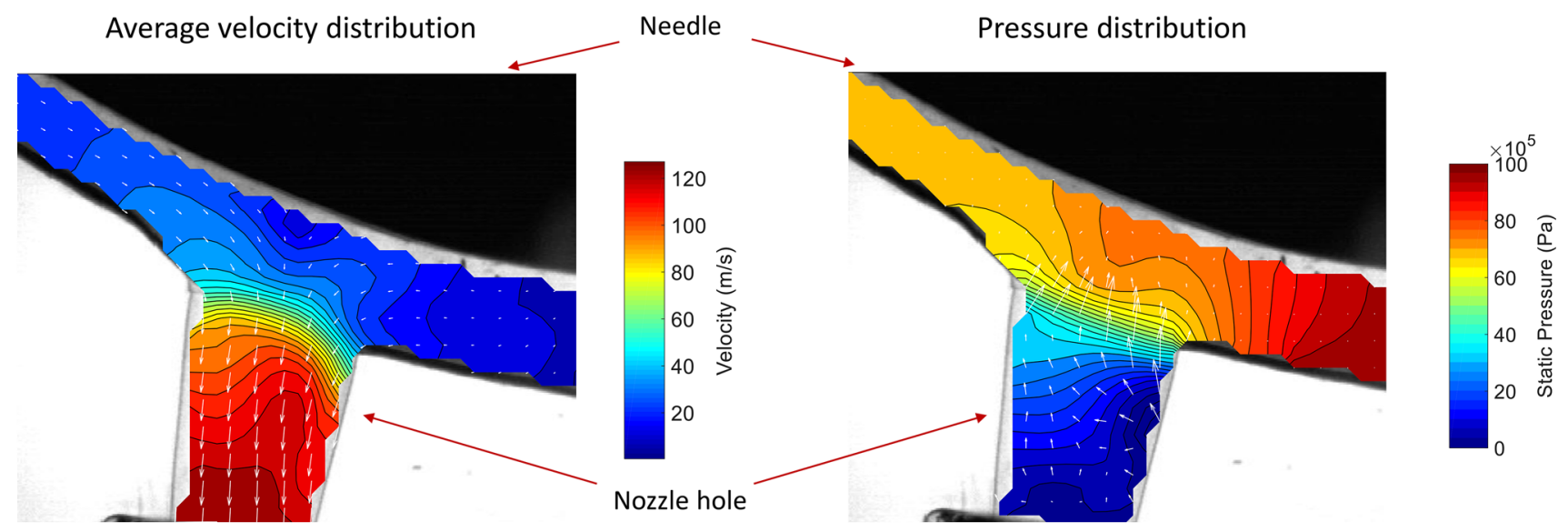

Dmitrii Mamaikin

dmitrii.mamaikin@fau.de

Extended author information available on the last page of the article 


\section{Introduction}

Modern combustion engines are typically equipped with direct injection systems. These systems are characterized by the fuel delivery method of injecting it directly into the combustion chamber. The fuel is introduced in the form of a spray, which has a large impact on the air fuel mixture preparation and subsequent combustion quality. A number of studies were dedicated to the investigation of spray properties and formation (Mitroglou et al. 2006; Moon et al. 2015; Knorsch et al. 2017). In regard to gasoline direct injection (GDI) applications, two major goals are typically of interest such as droplet size minimization and reduction of the spray penetration to avoid undesirable wall wetting.

It has been shown (Elbadawy et al. 2015; Postrioti et al. 2015) that spray performance depends on aerodynamic forces and ambient conditions. It is also known, however, that such characteristics as flow turbulence, velocity profile relaxation, cavitation, nozzle geometry and fuel properties are highly influencing factors to the jet breakup. The impact of the nozzle geometry has been studied intensively in recent years. As a result, researchers have observed variations in the spray performance for different designs. While consistent dependencies between spray properties and injector design parameters have been found, the details of how the design parameters affect the flow inside the nozzle and how this, in turn, affects spray properties remain unclear. Other works (Zigan et al. 2010; Henkel et al. 2017) have found that the physical properties and chemical composition of the fuel influences the spray formation process. The work of Schmitz et al. (2002) states that this effect is more pronounced for the conditions that induce the flash boiling phenomenon.

In recent times, many studies were focused on the analysis of cavitation. A number of numerical studies have been performed in relation to cavitation (Befrui et al. 2011; Zheng 2013; Saha et al. 2015). In turn, available experimental data is often based on large scale models (Aleiferis et al. 2006; Nouri et al. 2007; Kolokotronis et al. 2010). Some studies have achieved simultaneous observation of the internal flow and spray propagation. For example, Gavaises and Andriotis (2006) revealed the appearance of string cavitation inside nozzle holes. Moreover, the authors presented the impact of the string cavitation on the spray angle. Such studies on scaled models provide valuable insights into the fundamental in-nozzle flow behavior, however, the results often require plausibility checks to be transferred to practical flow conditions. Other experimental works performed the study of internal flow on real scale nozzles (Gilles-Birth et al. 2005; Reid et al. 2014; Moon et al. 2015; Mamaikin et al. 2017). Such studies provide a good insight into the cavitation behavior at relevant injection conditions. Andriotis et al. (2008) performed a comprehensive investigation of string cavitation. The authors state that string cavitation tends to occur in the areas of flow circulation. They also found that the string cavitation originates from the pre-existing cavitation sites, for example, from geometric-induced cavitation. Moreover, it can be initiated by outside air drawn into the nozzle hole as it was observed for convergent holes where the geometric-induced cavitation is inhibited. Thereby, these studies deliver a qualitative analysis of the cavitation behavior inside nozzles.

Quantitative results of the internal flow are still rare in literature. Walther et al. (2000) obtained velocity data of the internal flow in a transparent diesel nozzle by means of micro particle image velocimetry (PIV), using fluorescent particles. The results reveal a velocity field and flow lines inside a single hole diesel nozzle. Also, the technique applied in this study could visualize a recirculation zone inside the nozzle. Chaves et al. (2010) performed PIV measurements on a transparent single hole diesel nozzle. The injection pressure applied in the measurement is remarkably lower than typically used in diesel systems. The focus of the work, however, lies in the novel measurement technique for PIV, where graphite particles were used as a tracer. Kolokotronis et al. (2010) obtained velocity data by means of PIV for a large scale nozzle. The data shows valuable flow information and reveals three-dimensional flow in the nozzle. The cavitation similarity of the flow, however, was technically unreachable.

Pressure distribution in the flow is of special interest in the present work since it is a quantitative way to analyze the phenomenon of cavitation inception. Several research works were aimed to evaluate the pressure fields based on velocity data. Gurka et al. (1999) obtained velocity distribution by the PIV method and determined the steady pressure field in laminar pipe flow and impinging jet by solving the Poisson equation. The work of Baur and Köngeter (1999) presents the estimation of unsteady pressure fields of the flow including a wall-mounted obstacle using timeresolved data. Furthermore, some studies used the pressure data to determine the aerodynamic forces on objects like an aerofoil (van Oudheusden et al. 2007) or around a Savonius turbine (Murai et al. 2007).

A numerical investigation is a standard approach to evaluate the complex nature of the flow inside injector nozzles. Such investigations are realized by physical models and are typically based on some assumptions. The performance of numerical codes has been greatly improved in recent years. However, such models typically rely on experimental information, which is commonly used for validation or calibration purposes. Therefore, precise 
experimental data is vital for further improvements of advanced numerical codes.

The aim of this study is to experimentally characterize the internal flow under relevant injection conditions. 2D micro PIV measurements are conducted on a true scale transparent nozzle. The velocity data obtained is used to reconstruct the pressure distribution by applying the Reynolds-averaged Navier-Stokes (RANS) equations. Both data are compared with the predictions provided by a standard computational fluid dynamics (CFD) solver. Moreover, it is shown that the fluctuating terms of RANS can be employed to characterize the flow vorticity inside the nozzle hole. Subsequently, the proposed method might be used to estimate the transient phenomenon of string cavitation inside GDI nozzles.

\section{Experimental setup}

The experimental test bench configuration used in this study is depicted in Fig. 1a. The test bench was already used in the previous work published by Mamaikin et al. (2017), however, some modifications have been implemented to facilitate micro PIV measurements of the internal flow. Here, we briefly describe the main body and working principle of the test bench, placing an emphasis on the novel features. The experimental setup consists of fuel injection and imaging systems. The fuel is stored in a vessel, which is upstream to the modified Bosch HDEV5 gasoline injector. The vessel is connected to a high-pressure supply, which can deliver injection pressures up to 100 bar. The fuel temperature in the experiment is maintained at $25^{\circ} \mathrm{C}$, while the back pressure is set to $1 \mathrm{bar}$. The injector facilitates the mounting of transparent nozzles and enables dynamic needle operation. By that, visualization of transient nozzle flow is achieved. The desired maximum needle lift is precisely adjusted using spacer rings inside the injector body. The outlet container connects to the flushing line of the injector and the lowpressure nitrogen supply to support the possibility of back pressure variation. Before the start of the injection process, the nozzle holes are manually filled with fuel to eliminate air bubbles inside them. It enables optical access to the curved surfaces, and thereby to the flow, during the complete injection duration.

The test fuel selection is based on a few considerations. Among thermophysical properties, which are responsible for the flow behavior, the refractive index is an important parameter for optical experiments. The dissimilarity between the refractive indices of test fuel and acrylic glass causes optically inaccessible areas inside the nozzle due to its relatively complicated geometry with curved surfaces and the total reflection effect. Based on these considerations, the test fuel is represented by the calibration fluid (ISO 4113) with the addition of $18 \%$ by volume of 1 -Bromonaphthalene to match the refractive index of the acrylic glass nozzle (Walther 2002). It is noteworthy that some thermophysical properties of this mixture deviate from corresponding characteristics of gasoline, such as vapor pressure, density and particularly viscosity, which is in turn about ten times higher than that of gasoline. In this regard, the numerical work of Shi et al. (2010) investigated the effects of vapor pressure and viscosity on the internal flow behavior. As the authors stated, the vapor pressure has insignificant effect on cavitation when the operating temperature is well below that of the vaporization fuel temperature. This is due to the

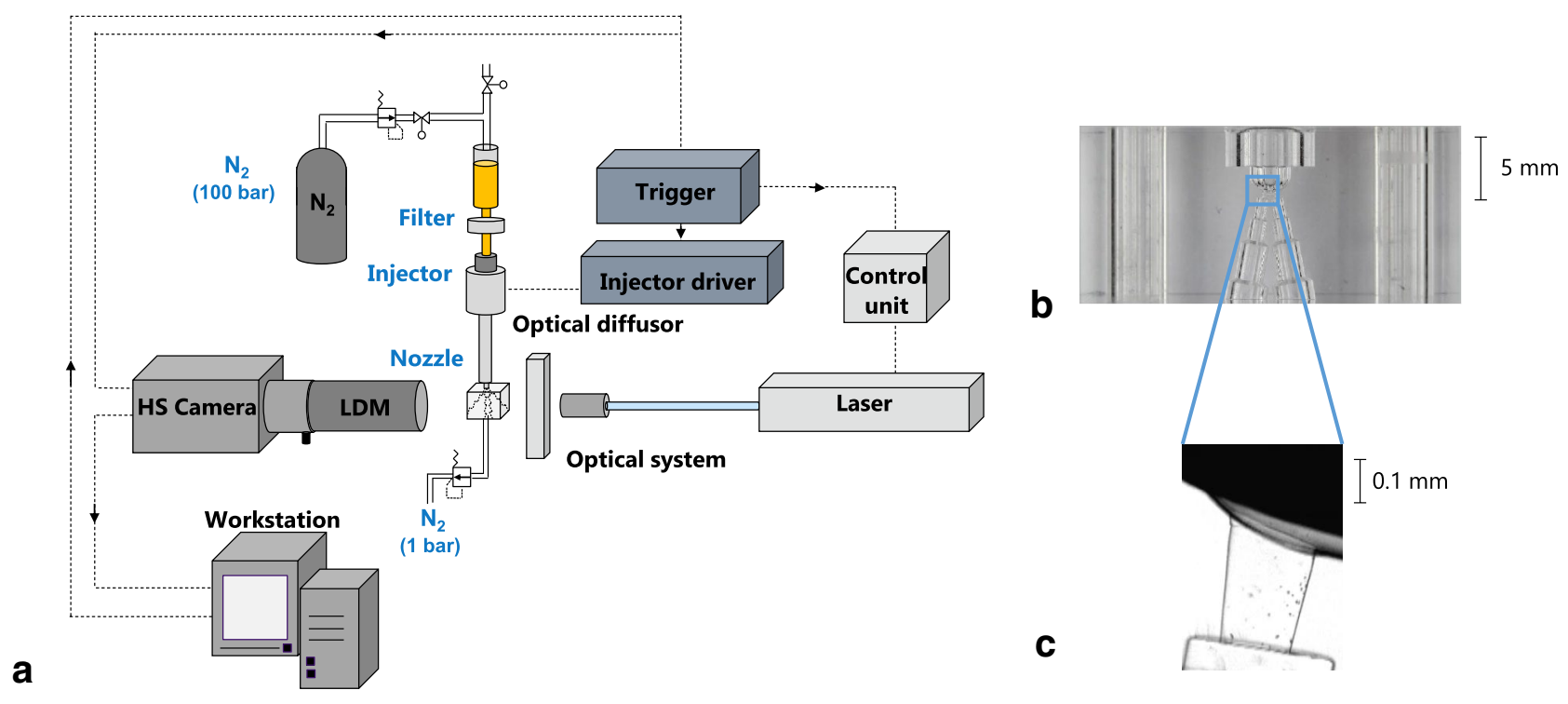

Fig. 1 a Experimental stand, $\mathbf{b}$ transparent nozzle and $\mathbf{c}$ shadowgraph image of the nozzle hole in a closed needle state 
high-pressure gradients in the nozzle. It is also mentioned that the fuel viscosity has a significant impact on the cavitation, and subsequently the internal nozzle flow. As a result, the optical access is prioritized to facilitate the experimental approach for the visualization of the transient cavitation phenomena with high optical quality and to enable velocity measurements inside a prototype GDI nozzle.

The transparent nozzle investigated in this study is shown in Fig. 1b. The nozzle has two holes, both bearing the true scale of the real injector nozzle and manufactured to be both on the same observation plane. The internal layout is represented as faithfully as possible taking into consideration mechanical robustness of the nozzle required for the experiment. Therefore, the nozzle is extended along the injector axis. The nozzle holes are followed by one counterbore of similar dimension to those used in series GDI nozzles and then by multiple larger counterbores that facilitate the withdrawal of the fuel, as it is already presented in the previous study published by Mamaikin et al. (2017). In this paper, the results of one nozzle hole are presented in detail. The nozzle hole of interest has a convergent shape and is designed to avoid any possible geometric-induced cavitation inside the hole. In preventing such cavitation, optical access inside the relevant region of the nozzle hole is maintained, which allows the possibility of a micro PIV investigation in this highly important area. However, the appearance of string cavitation in the nozzle hole is preserved due to the different nature of its formation. This tendency of the flow in a convergent hole was also observed for diesel nozzles as shown in Gavaises et al. (2009).

The PIV measurement realization by the conventional approach using a laser light sheet illumination is hardly applicable for the specific geometry of the experiment. Firstly, the tiny scales of the nozzle and extremely fast internal flow demand advanced and sophisticated imaging equipment to resolve dynamic flow characteristics. It is also crucial to obtain the transient evolution of the flow within a certain period for further pressure evaluation. Therefore, a high repetition rate of a light sheet illumination is required, which can hardly be achieved by the conventional lasers used for PIV. Secondly, 2D PIV approach in the current experimental design would imply that the light sheet enters the area of interest from the side of the nozzle to illuminate the flow in the plane, which is parallel to the one of the camera's image sensor. In that case, the hole edges and cavitation formations inside the nozzle holes may distort the light sheet path and subsequently lead to the erroneous interpretation of results. To overcome these challenges, the micro PIV approach using backlight illumination was chosen in this work. A novel imaging system has been implemented to meet the high temporal resolution required. The laser 'SILUX400' and ultra high-speed 'Kirana' camera fulfilled the required demand on the measurement technique. The camera is able to record 180 frames at a speed of $5 \mathrm{Mfps}$. The laser pulse duration is set to $10 \mathrm{~ns}$ to freeze the highly dynamic flow motion. The camera provides a resolution of $924 \times 768$ pixels. Thereby, this system provides the necessary spatial and temporal resolution, which is a favorable base for the study of highly dynamic multi-scale phenomena. Therefore, tracking of the particles is possible even inside the nozzle holes, which is commonly considered as a defining region for further liquid disintegration process. The velocity profile relaxation and initiation of cavitation are some of the most important data for numerical models. The experimental data in this crucial area barely exists, especially for transient high-pressure conditions.

The fuel has to be seeded with microparticles to visualize the flow for PIV evaluation. After analyzing a variety of potential seeding particles, graphite particles were selected as a tracer for this study. There are two main reasons for making this choice. Firstly, the opaqueness of the graphite particles facilitates the shadowgraph measurement approach, which is applied in this work. For example, polystyrene particles would also be a viable option unless their refractive index was to an extent identical to that of acrylic glass, which would cause these particles to be barely visible in images. Secondly, the soft graphite particles have low abrasive properties, meaning that acrylic glass nozzles will not sustain significant wear, thereby extending the lifetime of the transparent nozzles. For the same reason, this type of particle was also used in optical access engines, for example in the works of Neubert et al. (2011) and Buschbeck et al. (2012).

The particles have a mean diameter of $3.5 \mu \mathrm{m}$. It is noteworthy that the graphite particles have a density of $2.1 \mathrm{~g} / \mathrm{cm}^{3}$, which differs from that of the test fuel $0.94 \mathrm{~g} / \mathrm{cm}^{3}$. This difference causes the lag between the velocity of the liquid and the velocity of the following particle. However, this velocity lag can be estimated according to Stokes' law:

$U_{l}=\frac{\rho_{p}-\rho_{l}}{18 \mu} d^{2} a$

where $\rho_{p}$ is the particle density, $\rho_{l}$ is the fuel density, $\mu$ is the fuel dynamic viscosity, $d$ is the particle diameter and $a$ is the flow acceleration. The acceleration value is estimated based on the data from existing CFD results. According to this expression, the maximum expected velocity lag is 16 $\mathrm{m} / \mathrm{s}$ for the measurement conditions applied in the current study. This value can be considered as acceptable since the velocity range scales up to $150 \mathrm{~m} / \mathrm{s}$.

The long distance microscope (LDM) used was an Infinity K2. It is equipped with a CF4 lens, zoom module and NTX tube. This optical system allows for a magnification of 15.79. The depth of field (DoF) of the LDM is $28 \mu \mathrm{m}$. In addition, the concentration of seeding particles is another 


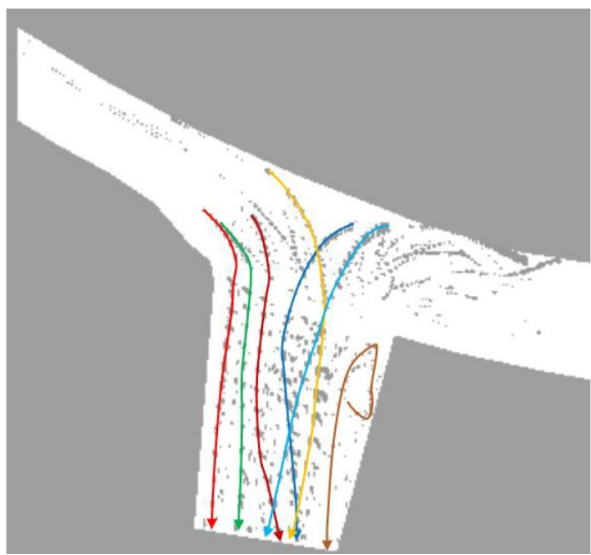

Fig. 2 Visualization of the flow lines by particle tracking

important aspect that defines whether individual particles or particle ensembles are evaluated as reported by Raffel et al. (2007). By this, the particle concentration is selected as $150 \mathrm{~g} / \mathrm{l}$ to facilitate the evaluation of the particle ensembles.

\section{Evaluation procedure}

\subsection{Qualitative flow analysis}

Initially, the qualitative analysis of the flow behavior is performed to capture the flow streamlines prior to the quantitative analysis using micro PIV evaluation. Figure 2 is obtained by combining a package of post-processed images, which is further used for the manual reconstruction of the flow lines. It should be noted that only those particles that are large and sharp enough are displayed and tracked over a relatively short time of about $8 \mu$ s to prevent a particle overlap on the image. The observation of streamlines provides an impression of the real three-dimensional flow even though the $2 \mathrm{D}$ imaging of the flow has been performed. The recirculation zone is detected on the up most part of the inner side of the hole for the time interval evaluated. Overall, the reconstructed streamlines reveal the swirling flow behavior inside the nozzle hole.

\subsection{Quantitative flow analysis}

\subsubsection{Internal velocity field}

The experimental settings along with accurate image preprocessing are relevant factors that might influence the quality of the data evaluated by means of micro PIV approach. After the discussion in Sect. 2, relevant settings have been summarized in Table 1 . The quality of raw images, in turn,
Table 1 Experimental settings for micro PIV evaluation

\begin{tabular}{ll}
\hline Temporal resolution & $0.2 \mu \mathrm{s}$ \\
Scale & $1.9 \mu \mathrm{m} / \mathrm{px}$ \\
Field of view & $924 \mathrm{px} \times 768 \mathrm{px}$ \\
Tracer mean diameter & $3.5 \mu \mathrm{m}$ \\
Pulse duration & $10 \mathrm{~ns}$ \\
\hline
\end{tabular}

might be reduced due to various undesirable technical aspects, such as inhomogeneous illumination, noise and blur effect. Under appropriate adjustment of the setup, these aspects can be minimized, however not eliminated. Therefore, careful image pre-processing is performed to achieve the best results from the micro PIV data evaluation. Thereafter, these images are used for the PIV evaluation algorithm. The pre-processing steps and successive PIV evaluation procedure are shown in Fig. 3. Initially, a mask is applied to the image to isolate the area of interest from the rest of an image to reduce data evaluation time. Then, bright field correction is applied to the image. This method of correction is based on the subtraction of the input image and the average image of three successive time series Gaussian filtered images. For further details regarding the bright field correction, refer to the product manual for $\mathrm{DaViS}$ (LaVision $\mathrm{GmbH}$ 2017). This step allows the removal of areas with constant intensity gradients, such as internal nozzle edges, improving the data accuracy. Finally, a Sobel filter is applied to sharpen the contours of the tracking particles. At this step, the image is fully pre-processed and ready for further PIV evaluation.

The pre-processed images are used for the velocity field estimation employing a state-of-the-art, cross-correlation algorithm. It is worth mentioning that a special method, so-called sliding sum-of-correlation (SOC) method, is used while evaluating the velocity fields. This method with a filter length of \pm 2 images (i.e. over 5 frames) is found to provide the best performance for the current measurement data and has two main benefits. Firstly, SOC achieves stable results by handling images with a lack of seeding particles, high image noise and high out of plane vector component correctly, imperfections which micro PIV images are often subjected to. Secondly, the sliding method allows maintaining the high temporal resolution of the resultant velocity data.

The particle image size range varies between 2 and 8 pixels. Maximum particle displacement between a pair of images under the framing applied is empirically measured as 14 pixels. This displacement corresponds to the maximum detectable velocity of $130 \mathrm{~m} / \mathrm{s}$, which has a high correlation with the velocity predicted by the Bernoulli equation considering 2D velocity field projection and the particle velocity lag mentioned in Sect. 2. The relevant evaluation settings for the velocity estimation are summarized in Table 2 . An interrogation area of $32 \times 32$ pixels with an overlap level of $75 \%$ 


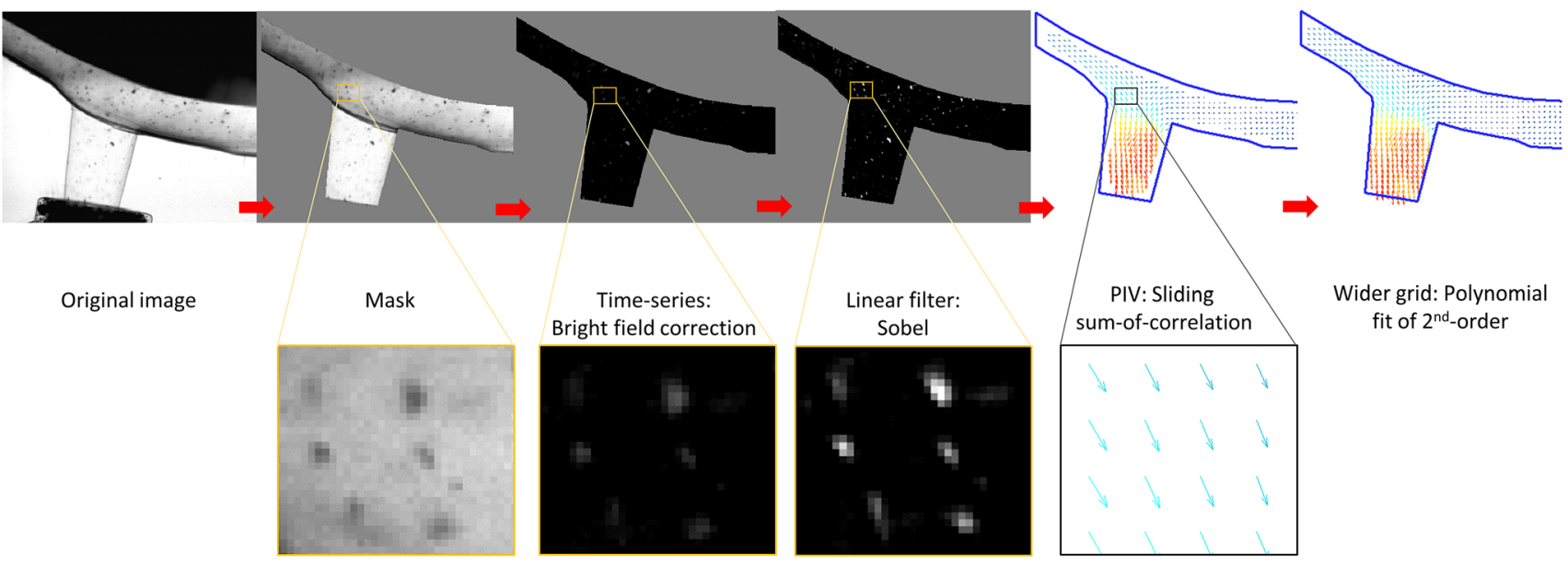

Fig. 3 Successive image processing steps for micro PIV data evaluation

Table 2 Micro PIV defining parameter settings

\begin{tabular}{ll}
\hline Filter length for PIV sliding SOC & \pm 2 images \\
Interrogation area & $32 \mathrm{px} \times 32 \mathrm{px}$ \\
Overlap & $75 \%$ \\
\hline
\end{tabular}

provides the spatial resolution of 8 pixels for the resultant velocity fields. Therefore, the spatial resolution is smaller for the velocity data due to the post-processing procedure. The reduction of spatial resolution is compensated by slight extrapolation of the velocity distribution to the boundaries using the polynomial fit of second-order, thereby slightly enlarging the area of the velocity field. This type of extrapolation leads to the overestimation of the velocities near walls. However, the larger area facilitates the pressure calculation procedure. As this study is mainly focused on the bulk flow field and string cavitation phenomenon, this slight extrapolation and subsequent errors near the walls are considered as acceptable. Larger nozzle holes or higher optical magnification, for instance using a microscope instead of LDM, could enlarge the flow areas with sufficient data gradients. In the current setup, the LDM was required due to the thickness of the glass nozzle.

\subsubsection{Pressure evaluation}

The reconstruction of the planar pressure field is a challenging task, especially when considering a turbulent threedimensional flow behavior, with 2D velocity information used as an input. Therefore, some assumptions are made to evaluate the pressure distribution inside the investigated nozzle at relevant injection conditions.

The micro PIV approach provides 2D velocity data, thus, the information about the third velocity component is not directly measurable. As stated by some CFD studies (for example, Zheng 2013), the flow inside nozzle holes does show rotational behavior. This flow behavior could also be circumstantially observed by streamlines obtained by particle tracking as shown in Fig. 2. When this rotating flow behavior is assumed, some information about the third velocity component can be returned as it will be shown further. For that, the transient flow characteristics are treated in a statistical sense by means of Reynolds decomposition (Adrian et al. 1998). Hereby, an instantaneous quantity is decomposed into its time-averaged and fluctuating quantities as shown below

$u(t, x, y, z)=\bar{u}(x, y, z)+u^{\prime}(t, x, y, z)$

$v(t, x, y, z)=\bar{v}(x, y, z)+v^{\prime}(t, x, y, z)$

$w(t, x, y, z)=\bar{w}(x, y, z)+w^{\prime}(t, x, y, z)$

$p(t, x, y, z)=\bar{p}(x, y, z)+p^{\prime}(t, x, y, z)$

where $u, v, w$ are the instantaneous velocity components in $x, y, z$ axes respectively and $p$ is the instantaneous static pressure. It is important to mention that, due to the principle of the PIV evaluation procedure, the flow is investigated in the Eulerian perspective.

These parameters can be directly implemented in Navier-Stokes equations. For the case of incompressible flows, the gradient $\frac{\partial \bar{w}}{\partial z}$ can be derived from the velocity gradients in the observation plane according to equation 6 , which is based on the continuity equation. The assumption of incompressible flow is reasonable while the Mach number of the flow is below the critical one $(M \ll 0.3)$ and 
data evaluation is applied for non-cavitating flow conditions.

$\frac{\partial \bar{w}}{\partial z}=-\left(\frac{\partial \bar{u}}{\partial x}+\frac{\partial \bar{v}}{\partial y}\right)$

Therefore, accounting for this contribution, the resulting RANS equations in the observation plane can be derived in the following form:

$$
\begin{aligned}
\frac{\partial \bar{p}}{\partial x}= & -\rho\left(\bar{u} \frac{\partial \bar{u}}{\partial x}+\bar{v} \frac{\partial \bar{u}}{\partial y}+\bar{w} \frac{\partial \bar{u}}{\partial z}\right) \\
& -\rho\left(\frac{\partial \overline{u^{\prime 2}}}{\partial x}+\frac{\partial \overline{u^{\prime} v^{\prime}}}{\partial y}+\frac{\partial \overline{u^{\prime} w^{\prime}}}{\partial z}\right) \\
& +\mu\left(\frac{\partial^{2} \bar{u}}{\partial x^{2}}+\frac{\partial^{2} \bar{u}}{\partial y^{2}}+\frac{\partial^{2} \bar{u}}{\partial z^{2}}\right) \\
\frac{\partial \bar{p}}{\partial y}= & -\rho\left(\bar{u} \frac{\partial \bar{v}}{\partial x}+\bar{v} \frac{\partial \bar{v}}{\partial y}+\bar{w} \frac{\partial \bar{v}}{\partial z}\right) \\
& -\rho\left(\frac{\partial \overline{u^{\prime} v^{\prime}}}{\partial x}+\frac{\partial \overline{v^{\prime 2}}}{\partial y}+\frac{\partial \overline{v^{\prime} w^{\prime}}}{\partial z}\right) \\
& +\mu\left(\frac{\partial^{2} \bar{v}}{\partial x^{2}}+\frac{\partial^{2} \bar{v}}{\partial y^{2}}+\frac{\partial^{2} \bar{v}}{\partial z^{2}}\right)
\end{aligned}
$$

Another appropriate representation of these equations is represented in Einstein notation as

$\frac{\partial \bar{p}}{\partial x_{k}}=-\rho \bar{u}_{l} \frac{\partial \overline{u_{k}}}{\partial x_{l}}-\rho \frac{\partial \overline{u_{k}^{\prime} u_{l}^{\prime}}}{\partial x_{l}}+\mu \frac{\partial^{2} \overline{u_{k}}}{\partial x_{l} \partial x_{l}}$

where $-\rho \overline{u_{l}} \frac{\partial \bar{u}_{k}}{\partial x_{l}}$ is the contribution of the mean momentum (Euler terms), $-\rho \frac{\partial \overline{u_{k}^{\prime} u_{l}^{\prime}}}{\partial x_{l}}$ is the Reynolds stress (fluctuating terms) and $\frac{\partial^{2} \overline{u_{k}}}{\partial x_{l} \partial x_{l}}$ corresponds to the viscous terms, $\rho$ corresponds to the density and $\mu$ to the dynamic viscosity. To facilitate pressure calculations, the out of plane velocity component $w$ and two components of the viscous friction terms $\frac{\partial^{2} \bar{u}}{\partial z^{2}}$ and $\frac{\partial^{2} \bar{v}}{\partial z^{2}}$ are assumed to be negligible. All the other terms are known from the experiment. Therefore, the mean pressure gradient can be derived directly from the velocity information. The mean and fluctuating velocity contributions are calculated according to the following equations:

$\overline{u_{k}}=\frac{1}{N} \sum_{i=1}^{N} u_{k}\left(t_{i}, x, y\right)$

$\overline{u_{k}^{\prime} u_{l}^{\prime}}=\frac{1}{N} \sum_{i=1}^{N} u_{k}^{\prime}\left(t_{i}, x, y\right) u_{l}^{\prime}\left(t_{i}, x, y\right)$

The contribution of Euler terms, Reynolds stress and viscous terms are further analyzed. The computed pressure gradient vectors are shown in Fig. 4. The vectors for Reynolds stress and viscous terms are magnified by a factor of 50 and 300 respectively. It can be concluded that the contribution from the viscous terms is negligible. The Euler terms, in turn, dominate in the flow, whereas the fluctuating terms are relevant for the turbulent area.

The actual pressure is obtained from the spatial integration of the Poisson equation (see Eq. 12) retrieved by the divergence of the pressure gradient neglecting the viscous terms.

$$
\frac{\partial^{2} \bar{p}}{\partial x_{k} \partial x_{k}}=-\rho\left(\frac{\partial \overline{u_{l}}}{\partial x_{k}} \frac{\partial \overline{u_{k}}}{\partial x_{l}}+\overline{u_{l}} \frac{\partial^{2} \overline{u_{k}}}{\partial x_{k} \partial x_{l}}\right)-\rho \frac{\partial^{2} \overline{u_{k}^{\prime} u_{l}^{\prime}}}{\partial x_{k} \partial x_{l}}
$$

\subsubsection{Iterative pressure integration}

All parameters in Eq. 12 are known except for the pressure field. In mathematical terms, this equation represents a second-order nonlinear elliptic partial differential equation. To find the pressure, the equation has to be discretized in an algebraic finite difference form. The time-resolved velocity vector field obtained by the PIV measurements has already been discretized in space with the steps $\Delta x$ and $\Delta y$ in $x$ and $y$ directions respectively. Every point of the grid can be derived by introducing the integer index $i$ and $j(x=i \Delta x$, $y=j \Delta y$ ). By applying the central difference scheme to calculate the pressure value at the point $(i, j)$, the Poisson equation is transformed according to Eq. 13:

$\overline{p_{i, j}}=\frac{\left(\overline{p_{i+1, j}}+\overline{p_{i-1, j}}\right) \Delta y^{2}+\left(\overline{p_{i, j+1}}+\overline{p_{i, j-1}}\right) \Delta x^{2}-b_{i, j} \Delta x^{2} \Delta y^{2}}{2\left(\Delta x^{2}+\Delta y^{2}\right)}$

where $b_{i, j}$ is the known right-hand side element of Eq. 12 at the point $(i, j)$.

The grid domain is complex due to its non-rectangular shape, therefore an appropriate way to integrate the pressure is to use iterative methods. Thereby, the Poisson equation can be solved iteratively when the initial estimate for the pressure field is provided. For this purpose, the successive over-relaxation (SOC) iterative method is used in this work. Boundary conditions are defined on all edges of the evaluation domain. The point is considered as an edge when at least one velocity data, compared to 8 surrounding points, is missing. Neumann boundary conditions (pressure gradient is prescribed) are enforced using the RANS equations (Eq. 9). Additionally, a reference boundary condition has to be fixed (pressure is prescribed). Ideally, this condition should be defined in the outer flow, where the Bernoulli equation can be applied. In the present experiment, it has to be fixed in the nozzle flow due to the limited observation domain into the flow. De Kat and van Oudheusden (2011) introduced the extended version of Bernoulli equation to correct for this 
Fig. 4 Pressure gradient distribution for total and individual contributing terms
Mean pressure gradient - total

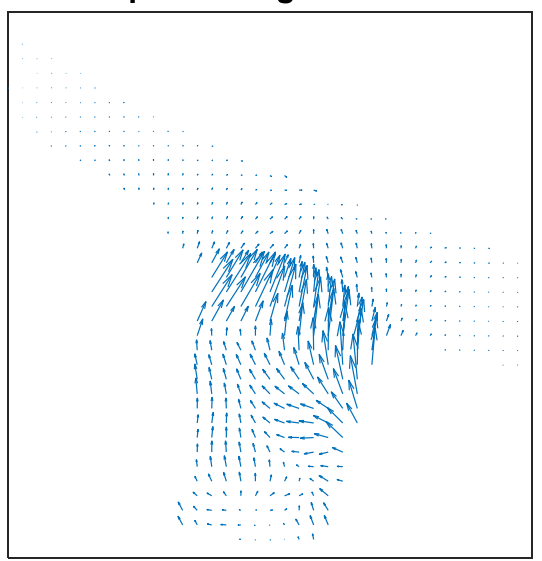

Fluctuating terms (50x magnified)

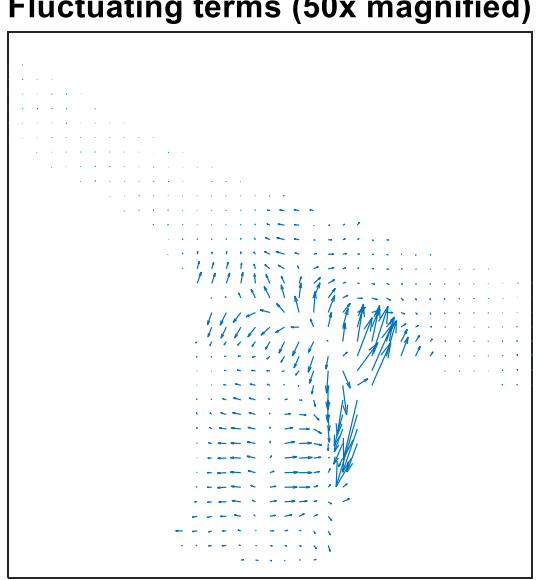

Euler terms

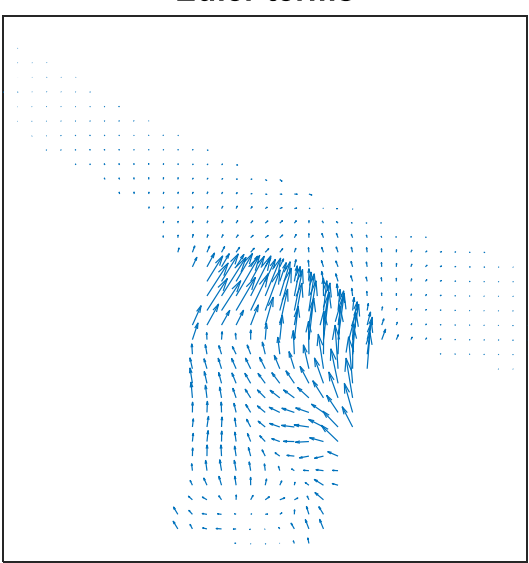

Viscous terms (300x magnified)

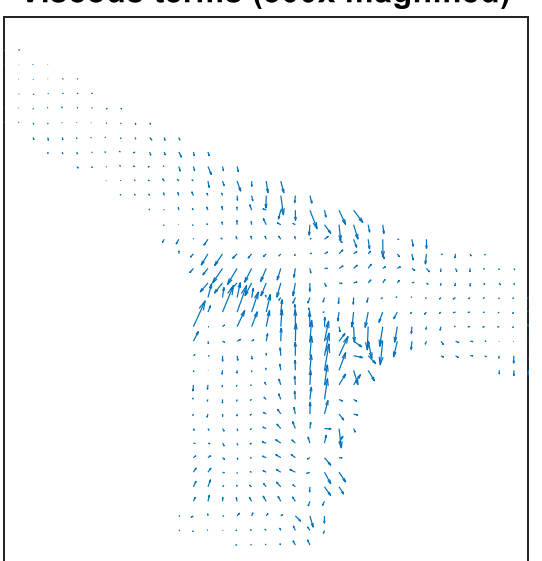

type of issue. In a similar way, the extended Bernoulli equation is also used in this work to define the reference pressure that holds for an irrotational flow with small mean velocity gradients. This equation can be represented as shown below

$\bar{p}+\frac{1}{2} \rho\left(\bar{u}^{2}+\bar{v}^{2}+\overline{u^{\prime 2}}+\overline{v^{\prime 2}}\right)=p_{\infty}+\frac{1}{2} \rho V_{\infty}^{2}$

where $p_{\infty}$ and $V_{\infty}$ are the injection pressure and the velocity magnitude in the upstream flow respectively, which can be reasonably neglected as the flow in the injector body is very slow.

\section{Numerical model}

A numerical study is performed to compare and thoroughly discuss the velocity data and pressure distribution obtained in the framework of the current experimental investigation. The software ANSYS CFX 19.2 has been used for flow modeling. The nozzle flow simulations typically consist of three phases, namely liquid fuel, vapor and air. Mithun et al. (2018) stated that the combination of these phases is essential to be introduced in case the link between the internal flow and primary atomization is numerically investigated. Therefore, the internal nozzle flow is modeled by a homogeneous mixture of these phases. The transient flow is described by an unsteady Reynolds-averaged Navier-Stokes (URANS) approach using the shear stress transport (SST) turbulence model. The cavitation is incorporated by a Rayleigh-Plesset equation-based model. The nozzle layout is axis-symmetrical. A $180^{\circ}$-sector geometrical model is adopted and a symmetry boundary condition is used on segment surfaces. A static pressure opening condition was defined in the model. The pressure and temperature boundary conditions are defined to be the same as in the experiment. As a major component, the properties of calibration fuel (ISO 4113) are used for the flow simulation. The mesh constitutes roughly 4 million cells in the model. The temporal resolution is $0.1 \mu \mathrm{s}$. The flow is solved on the Eulerian frame of reference. 


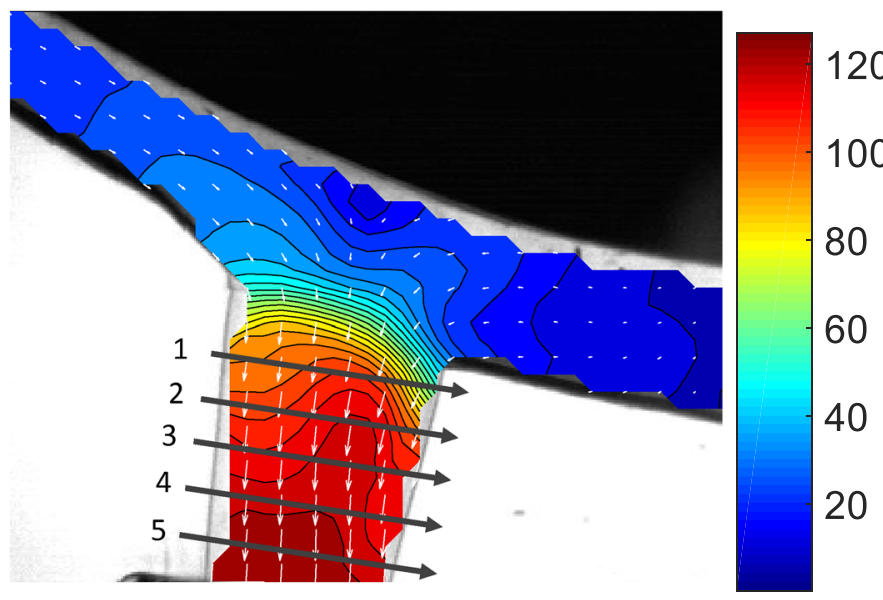

120

100

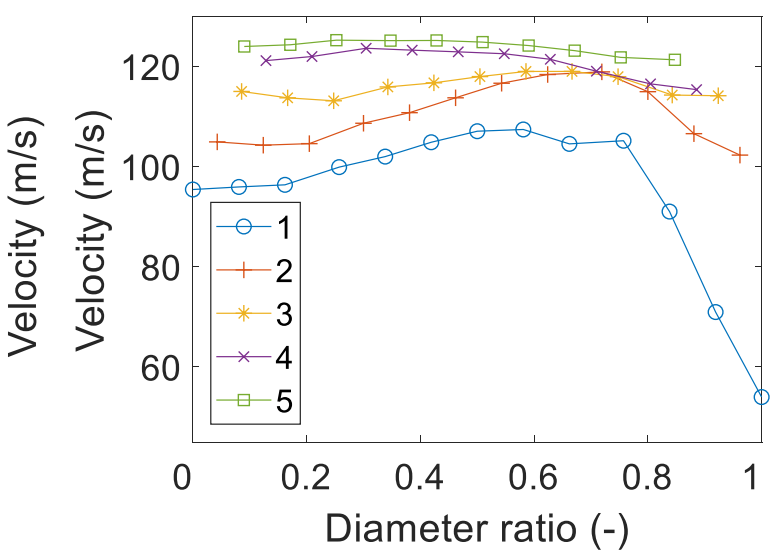

Fig. 5 Mean velocity and velocity profiles

\section{Results and discussion}

\subsection{Velocity and pressure fields}

Firstly, the experimental results are discussed. Figure 5 shows the velocity field obtained by the micro PIV evaluation procedure of the nozzle flow in a steady-state injection phase at the injection pressure of 100 bar. The mean data is based on the evaluation of 156 single images. The velocity magnitude is shown as a field in false color and velocity direction is shown with white arrows. Both of them are plotted on the nozzle image, which serves as a background.

It can be seen that the flow velocity gradient increases when entering the nozzle hole. A broad velocity range is detected in the nozzle, varying from approximately $10 \mathrm{~m} / \mathrm{s}$ to $130 \mathrm{~m} / \mathrm{s}$. The maximum velocity, according to the Bernoulli equation, is expected to approach $150 \mathrm{~m} / \mathrm{s}$. Therefore, the measurement data obtained in this study is in the

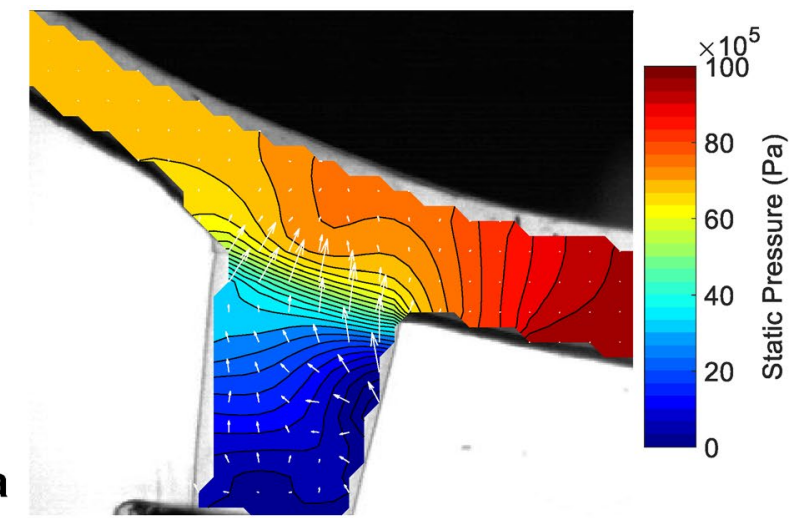

expected range, considering the $2 \mathrm{D}$ projection of the real viscid flow. The velocity profile relaxation along the hole axis is also shown in Fig. 5. The velocity profiles correspond to the appropriate cross-sections depicted in the image of mean velocity distribution. It can be seen that the maximum velocity values take place marginally towards the outer side of the injector. In other words, the asymmetric velocity distribution inside the hole is detected. It is important to note that no negative velocity values are obtained in contrast to the results shown in Fig. 2, where a small vortex is observed on the upper right side of the hole by means of particle tracking. The reason for that is the difference in the time intervals used for these evaluation strategies. The period used to obtain the PIV data is almost four times longer than the one used for particle tracking. Therefore, the average velocities are smoothed over the whole evaluation time. Nonetheless, the influence of the short-term vortex is observable on the velocity profiles shown in Fig. 5, where the velocity values drop

Fig. 6 a Pressure distribution and $\mathbf{b}$ contribution of fluctuation 
drastically in the hole area corresponding to the vortex location. Overall, it is clear that the velocity magnitude increases along the axis of the hole. This phenomenon is expected, due to the convergent hole geometry.

The pressure distribution, derived from the measurement data by means of RANS, is shown in Fig. 6a. The positive pressure gradients are indicated by white arrows on the image. The overall pressure range varies from 2 bar to $100 \mathrm{bar}$, which serves as the initial boundary condition. The pressure upstream of the nozzle hole reveals a different ratio on either side of the hole. Furthermore, the pressure on the outer side of the injector is lower than the inner by approximately 30 bar. The outer side is geometrically closer to the needle seat, which is a throttle before the hole inlet. Thus, the flow on the outer side of the injector is subjected to a higher negative pressure gradient compared to the inner side. The minimum pressure is located in the nozzle hole, particularly on the inner side of the injector. This phenomenon is a result of higher flow deflection on this side when entering the nozzle hole.

Overall, the static pressure distribution is derived from 151 successive velocity fields. Thereby, it provides valuable information to increase knowledge of the internal flow phenomena. As described in Sect. 3.2, the result is based on the $2 \mathrm{D}$ velocity information. However, it is known that the string cavitation is the result of the three-dimensional flow structure. In further analysis, the fluctuating terms of the RANS equations are used to evaluate the swirling degree of the flow. As it is mentioned earlier, some CFD studies state that string cavitation is a result of strong rotational flow (Zheng 2013). In terms of 2D velocity measurement, such a rotating flow leads to the fluctuation
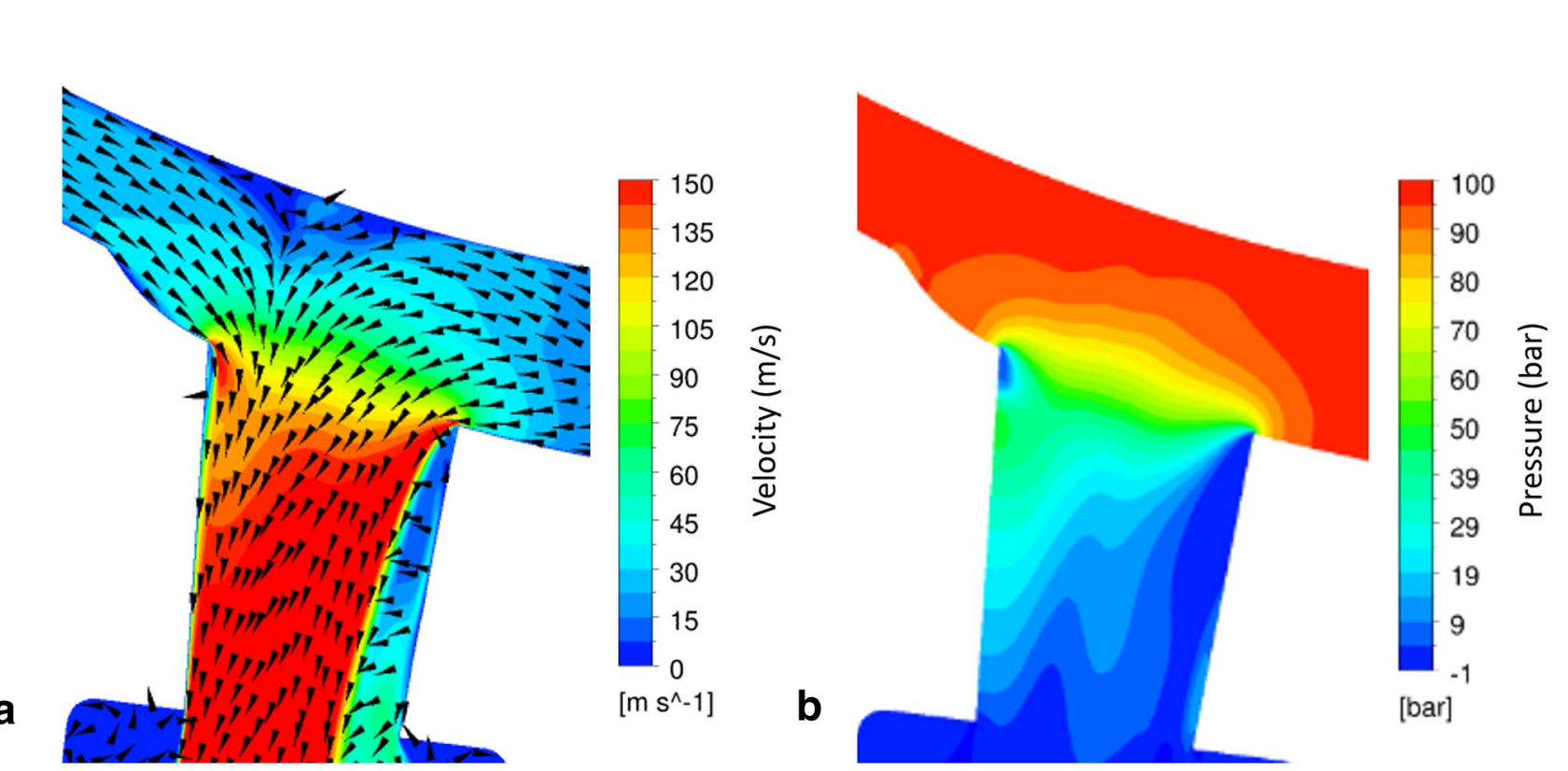

Fig. 7 a Velocity and $\mathbf{b}$ pressure distributions obtained by CFD of velocity direction at observation locations over time. The prerequisites to the measurement equipment needed for this evaluation are sufficient time resolution to detect velocity fluctuations and the combination of the appropriate magnitude of DoF with the sufficient particle size that allows the tracking of seeding particles in the range of the radial dimension of rotating flow structure. Both requirements are fulfilled in the current study.

The fluctuating terms contribution to the static pressure is presented in Fig. 6b which means $P_{\text {stat }}=B C+\Delta P_{\text {fluc }}+\Delta P_{\text {Euler }}$ with $\mathrm{BC}$ the fixed value set on the boundary, $\Delta P_{\text {fluc }}$ the contribution from the fluctuating terms to the static pressure, $\Delta P_{\text {Euler }}$ the contribution from the Euler terms to the static pressure. The results are presented in difference form of pressure fluctuation and are shown in false color. The distribution reveals two main fluctuating regions. The first region takes place in the lower central part of the nozzle hole. This region corresponds to the place where the flow is highly rotating, which indirectly might indicate the probability of string cavitation. The second fluctuating area inside the nozzle hole is on the upper part of the inner side of the injector. This location is subjected to the recirculation area of the flow due to high flow deflection. This area was already identified by the flow lines in Fig. 2. Therefore, the regions of fluctuation refer to the nozzle areas that are potentially subjected to the cavitation phenomenon for this specific nozzle shape. It is important to note that neither string nor film cavitation was observable during the flow visualization period evaluated in this section, otherwise, no PIV data could have been obtained. As a result, this evaluation approach inside cavitation free nozzle holes can be used 
to determine and qualitatively estimate the swirling flow structures.

For comparison, the velocity distribution obtained from the numerical study is presented in Fig. 7a. It can be seen that the CFD study expects flow separation on the inner side of the injector. The experimental results shown in Fig. 5a do not show this flow behavior, at least in the observation time of $36 \mu \mathrm{s}$, which has been evaluated. This difference could be influenced by the fuel properties, where only a major component of the test fuel mixture was used for the CFD study as mentioned in Sect. 4. On a more positive note, it was shown by the particle tracking (see Fig. 2) that the short term flow circulation was observed in the corresponding hole area. However, as discussed earlier, the velocity distribution does not reveal distinct recirculation flow behavior in this region because of the averaging over a longer injection period. The absolute velocity values obtained in the experiment are slightly underestimated in comparison to the results provided by the CFD study. This difference can be explained by the smaller effective diameter of the hole in the case of CFD due to the flow separation. For that case, it has been shown that the reduction of the effective hole diameter can lead to an increase in the outlet velocity (Payri et al. 2004). Furthermore, the velocity lag between flow and seeding particles also contributes to the velocity underestimation in the experiment as mentioned in Sect. 2. Therefore, except for the flow separation area, the flow results obtained by the CFD study reveal comparable velocity distribution in the nozzle. A relatively slow flow region located right upstream of the hole is found in both experimental and numerical results. Moreover, the flow on the outer side of this area is found to be faster due to the region being next to the needle seat. The flow acceleration along the hole axis can be observed, which is in a fair agreement between both cases.

Figure $7 \mathrm{~b}$ shows the pressure distribution obtained by the simulation. It can be observed that the pressure field inside the nozzle hole reveals similar distribution as the pressure results obtained based on the experimental data. Both cases reveal that the lowest pressure area in the hole is located on the inner side of the injector. Therefore, a rather high pressure gradient is found on the inner side, whereas the pressure gradient is smaller to some extent on the outer side of the injector. Another low-pressure region is found in the hole exit in the area between the hole axis and the outer side of the injector. This observation can also be noticed in the pressure evaluation based on the experimental data. In contrast, the area upstream of the hole reveals some differences in the pressure distribution. The main reason for that is the elevated 3D flow effects and a relatively small area for the pressure integration based on the 2D experimental data. Overall, PIVbased pressure evaluation of turbulent flows is highly challenging due to a large dynamic range and small magnitude of pressure fluctuations. As a consequence, some works exhibit a relatively high discrepancy between the PIV-based estimated pressure and measured pressure (Ghaemi et al. 2012; Pröbsting et al. 2013). In the current work, the internal flow reveals even higher challenges due to the remarkably higher Reynolds number of the flow, micro-scale visualization and swirling flow behavior. Therefore, the pressure data is not recommended to be considered as reference data. For that, the CFD data could probably provide better pressure prediction, in case the numerical model is validated experimentally by other flow characteristics, e.g. velocity distribution and cavitation formations.

\subsection{String cavitation development}

As mentioned, the results presented in the previous section refer to the steady-state injection phase. In this section, the results that correspond to the transient needle phase are discussed. It was found that the moving needle provokes the initiation of string cavitation. This observation may be linked to a smaller needle lift during the needle opening or closing and therefore a more asymmetrical flow inside the nozzle hole, or perhaps the more turbulent flow from a change in the throttling position. In the present study, the evaluation of the flow with a transient injection period is undertaken. Particularly, the nozzle flow during the needle closing phase is shown. The measurement data evaluated in this section corresponds to the injection pressure of $50 \mathrm{bar}$. All the other conditions remain identical to the rest of the paper. This change is related to the sufficient data without string cavitation for the micro PIV evaluation and the data including the string cavitation at the same recording event. The results for fluctuation evaluation are shown in Fig. 8. It can be seen that string cavitation is expected to occur inside the hole with some left bias towards the outer side.

The successive images that follow after a short time, the images evaluated above, are presented further. The initiation of string cavitation is visible in Fig. 9. The time step between successive images is $0.2 \mu \mathrm{s}$. Initially, the cavitation

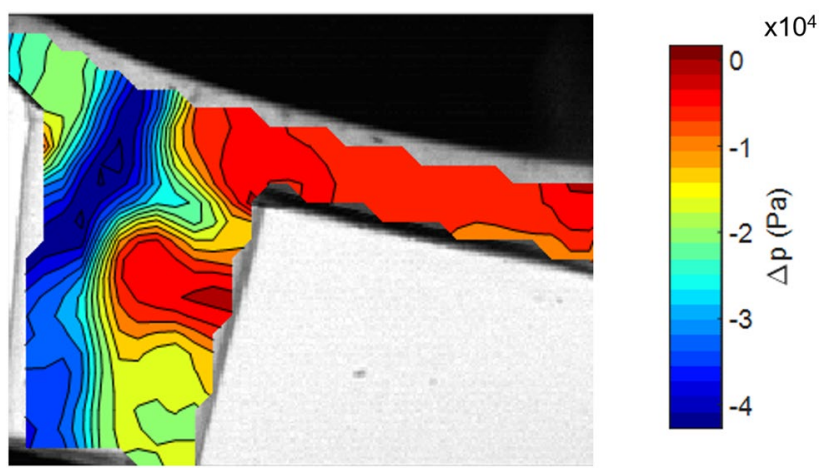

Fig. 8 Fluctuation terms for needle closing phase. Injection pressure 50 bar 

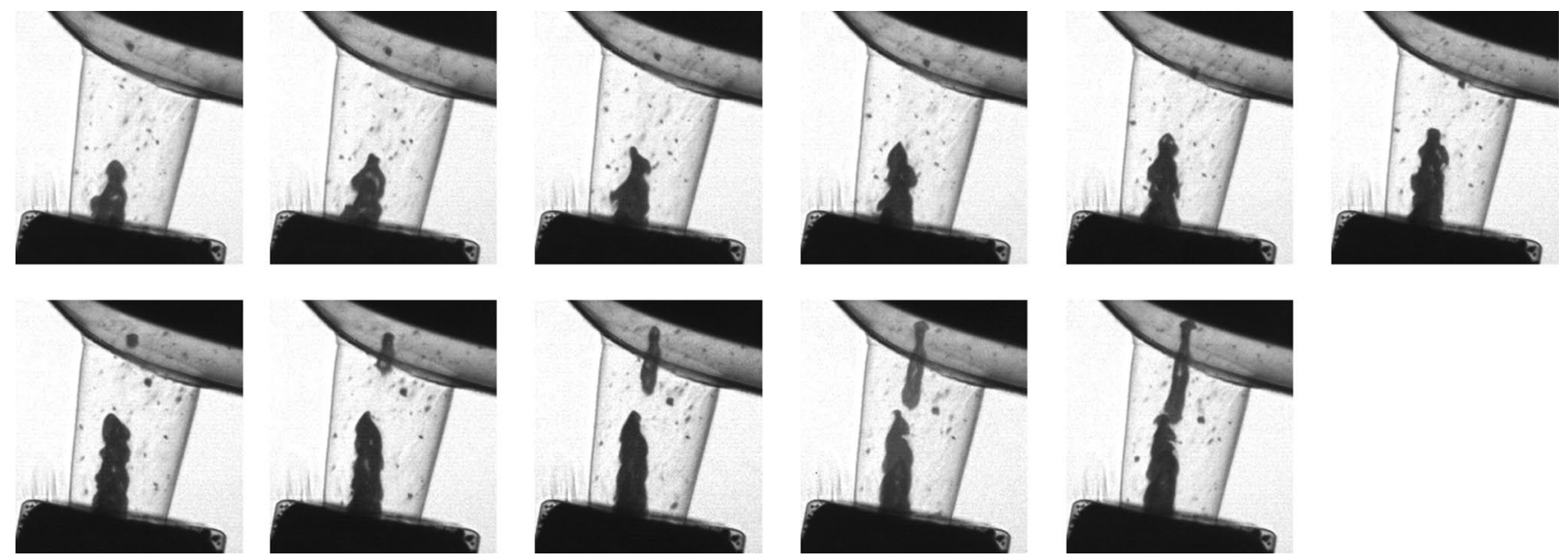

Fig. 9 Initiation of string cavitation

exists only in the lower part of the hole. As the needle closes further, this leads to higher flow vorticity, which results in the growth of such string cavitation. At some point, the initiation of the second origin of string cavitation is visible. This origin takes place between the needle and the entrance to the hole and it extends further into the hole. In a similar way, the initiation and development of string cavitation were observed by Roth et al. (2002) on the large scale diesel nozzle. They refer to this phenomenon as needle strings. As can be seen further, it develops quickly in both directions along the axis of the hole, merging with the initial string cavitation. By that, the cavitation extends from the near needle area to the hole exit. The dynamics of the cavitation present here, correlate with the fluctuating regions shown in Fig. 8 with a small shift to the left side until the border of the evaluation area. This shift could be explained by the slight extrapolation applied to the velocity distribution described in Sect. 3. Interestingly, the area of the second origin of the cavitation is also more pronounced in the distribution.

As observed, the string cavitation developed by the connection of two structures from different origins, revealing new insight into the analysis of the internal flow. However, this observation does not indicate that the string cavitation always develops in this manner, instead, it is likely to be more dependent on the nozzle hole geometry and the needle lift as in the case for geometric-induced types of cavitation.

Karathanassis et al. (2018) visualized the vortex cavitation in the nozzle applying the X-ray phase-contrast imaging method and used the data to calibrate the vortex model. In the same way, available vortex flow models are used to estimate the tangential velocity of the flow in the hole where the string cavitation appears. For that, the dimension of the string cavitation observed in Fig. 8 and corresponding flow conditions are employed in Gaussian and Lamb-Oseen vortex models.
The recirculation intensity $\mathrm{L}$ is found based on the following equation:

where $p_{c}$ and $p_{0}$ are the pressure at the vortex core and pressure in the free stream flow in absence of the vortex, $\rho$ corresponds to the fuel density and $r_{c}$ refers to the vortex core radius. The flow in the current type of measurements is quite transient and does not correspond to the continuous flow circulation. The pressure $p_{0}$ is assumed to be 10 bar as the pressure value representative to the pressure gradient in the orifice.

Based on that, the tangential velocity can be estimated under the assumption that it can be described by the Gaussian profile.

Single-phase Gaussian vortex:

$U_{\theta}=\frac{L}{2 \pi r}\left(1-\exp \left[-a\left(\frac{r}{r_{c}}\right)^{2}\right]\right)$

Cavitating Lamb-Oseen vortex (Pennings et al. 2015):

$U_{\theta}=\frac{L}{2 \pi r}\left(1-b \exp \left(-\mu \frac{r^{2}-r_{b}^{2}}{r_{c}^{2}}\right)\right)$

where $\mu=1.2654, a=1.255, r_{b}$ is the radius of a vapor core and $b=\frac{r_{c}^{2}}{r_{c}^{2}+\mu r_{b}^{2}}$. The core radius of the vortex $r_{c}$ is approximated as $\sqrt{2} r_{b}$ based on the conclusion of (Arndt and Keller 1992).

Figure 10 shows the results obtained by the two selected models. The maximum tangential velocity is estimated to be in the vortex core radius. This data provides supplementary $\frac{p_{c}-p_{0}}{\frac{1}{2} \rho\left(\frac{L}{2 \pi r_{c}}\right)^{2}}=1.74$ 


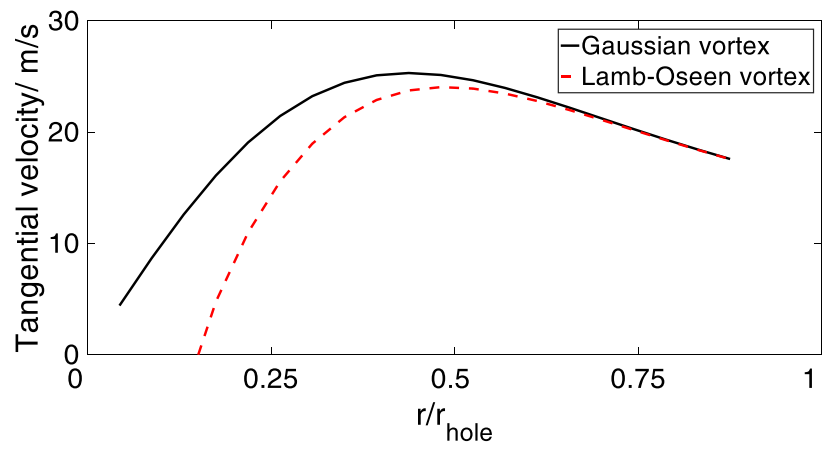

Fig. 10 Tangential velocity predicted using Eqs. 16 and 17

information for the volumetric velocity estimation of the flow in the nozzle hole. Important to note that the tangential velocity data is based on the experimental images with vortex cavitation, whereas the $2 \mathrm{D}$ PIV velocity data are obtained from the images before the vortex cavitation appears. In addition to this discrepancy in the evaluation time points, it is known that the flow characteristics can be altered once the cavitation occurs (Arndt 2002). Therefore, these aspects should be certainly taken into account if the estimation of volumetric velocity is desired.

\section{Conclusion}

Micro PIV measurements were performed for a transparent GDI nozzle using the true scale. The technical difficulties of this measurement technique were overcome by implementing novel, ultra high-speed imaging equipment. Thereby, the velocity field was acquired at relevant injection conditions inside the nozzle. The flow accelerates along the axis of the hole, a conclusion which was to be expected due to the convergent hole shape and the convergent design of the nozzle area between the bottom of the needle seat and the nozzle hole entrance. The velocity field upstream of the hole reveals a slightly faster flow on the outer side of the injector due to the impact of throttling in the needle seat. Additionally, the velocity data is further used for pressure evaluation within the internal nozzle domain. The static pressure distribution shows the minimum pressure region in the upper, inner side of the hole. This region was observed to be the recirculation area of the flow due to relatively high, local flow deflection. It is also shown that the analysis of the fluctuating terms of RANS provides valuable information regarding the swirling flow structures inside the nozzle. The evaluation presented in this paper is a unique approach to qualitatively analyze the rotating structures inside injector nozzles experimentally. By that, a better insight into the 3D flow structure, through means of a 2D measurement technique, is achieved.
Acknowledgements Open Access funding provided by Projekt DEAL. The authors would like to express their gratitude to the great assistance of Patrick Kajsztura, Loic Marfing and Manjunatha Siddaramanna. This work could not have been completed without their kind support.

Open Access This article is licensed under a Creative Commons Attribution 4.0 International License, which permits use, sharing, adaptation, distribution and reproduction in any medium or format, as long as you give appropriate credit to the original author(s) and the source, provide a link to the Creative Commons licence, and indicate if changes were made. The images or other third party material in this article are included in the article's Creative Commons licence, unless indicated otherwise in a credit line to the material. If material is not included in the article's Creative Commons licence and your intended use is not permitted by statutory regulation or exceeds the permitted use, you will need to obtain permission directly from the copyright holder. To view a copy of this licence, visit http://creativecommons.org/licenses/by/4.0/.

\section{References}

Adrian RJ, Christensen KT, Soloff SM, Meinhart CD, Liu ZC (1998) Decomposition of turbulent fields and visualization of vortices. In: 9th Int Symp Appl Laser Techniques Fluid Mech, Lisbon, Portugal, 13-16 July, pp 16.1.1-16.1.8

Aleiferis PG, Hardalupas Y, Kolokotronis D, Taylor AMKP, Arioka A, Saito M (2006) Experimental investigation of the internal flow field of a model gasoline injector using microparticle image velocimetry. SAE Technical Paper 2006-01-3374. https://doi. org/10.4271/2006-01-3374

Andriotis A, Gavaises M, Arcoumanis C (2008) Vortex flow and cavitation in diesel injector nozzles. J Fluid Mech 610:195-215

Arndt REA, Keller AP (1992) Water quality effects on cavitation inception in a trailing vortex. J Fluid Eng 114(3):430-438

Arndt REA (2002) Cavitation of vortical flows. Annu Rev Fluid Mech $34: 143-175$

Baur T, Köngeter J (1999) PIV with high temporal resolution for the determination of local pressure reductions from coherent turbulence phenomena. In: 3rd Int Workshop Particle Image Velocimetry, Santa Barbara, USA, 16-18 September, pp 101-6

Befrui B, Corbinelli G, D'Onofrio M, Varble D (2011) GDI multi hole injector internal flow and spray analysis. SAE International 201101-1211. https://doi.org/10.4271/2011-01-1211

Buschbeck M, Bittner N, Halfmann T, Arndt S (2012) Dependence of combustion dynamics in a gasoline engine upon the in-cylinder flow field, determined by high-speed PIV. Exp Fluids 53:17011712. https://doi.org/10.1007/s00348-012-1384-3

Chaves H, Eberle A, Hofemeier P (2010) Micro PIV for high velocity flows. In: 15th Int Symp Appl Laser Techniques Fluid Mech, Lisbon, Portugal, 05-08 July, pp 1-8

de Kat R, van Oudheusden BW (2011) Instantaneous planar pressure determination from PIV in turbulent flow. Exp Fluids 52:1089-1106

Elbadawy I, Gaskell PH, Lawes M, Thompson HM (2015) Numerical investigation of the effect of ambient turbulence on pressure swirl spray characteristics. Int J Multiphas Flow 77:271-284

Gavaises M, Andriotis A (2006) Cavitation inside multi-hole injectors for large diesel engines and its effect on the near-nozzle spray structure. SAE World Congress 2006-01-1114. https://doi. org/10.4271/2006-01-1114

Gavaises M, Andriotis A, Papoulias D, Mitroglou N, Theodorakakos A (2009) Characterization of string cavitation in large-scale Diesel nozzles with tapered holes. Phys Fluids 21:052107 
Gilles-Birth I, Bernhardt S, Spicher U, Rechs M (2005) A study of valve covered orifice nozzles for gasoline direct injection. SAE Technical Paper 200501-3684. https://doi.org/10.4271/2005-01-3684

Ghaemi S, Ragni D, Scarano F (2012) Turbulent structure of highamplitude pressure peaks within the turbulent boundary layer. Exp Fluids 53(6): 1823-1840

Gurka R, Liberzon A, Hefetz D, Rubinstein D, Shavit U (1999) Computation of pressure distribution using PIV velocity data. In: 3rd Int Workshop Particle Image Velocimetry, Santa Barbara, USA, 16-18 September, pp 671-676

Henkel S, Hardalupas Y, Taylor A, Conifer C, Cracknell R, Goh TK, Reinicke P, Sens M, Rieß M (2017) Injector foiling and its impact on engine emissions and spray characteristics in gasoline direct injection engines. SAE Int J Fuel Lubr. https://doi. org/10.4271/2017-01-0808

Karathanassis IK, Koukouvinis P, Kontolatis E, Lee Z, Wang J, Mitroglou N, Gavaises M (2018) High-speed visualization of vortical cavitation using synchrotron radiation. J Fluid Mech 838:148-164

Knorsch T, Mamaikin D, Leick P, Rogler P, Wang J, Li Z, Wensing M (2017) Comparison of shadowgraph imaging, phase Doppler anemometry and X-ray imaging for the analysis of near nozzle velocities of GDI fuel injectors. SAE Technical Paper 2017-012302. https://doi.org/10.4271/2017-01-2302

Kolokotronis D, Hardalupas Y, Taylor AMKP, Aleiferis PG, Arioka A, Saito M (2010) Experimental investigation of cavitation in gasoline injectors. SAE International 2010-01-1500. https://doi. org/10.4271/2010-01-1500

LaVision GmbH (2017) 1003005_FlowMaster_D84.pdf (ProductManual for DaVis 8.4., 1105011-4)

Mamaikin D, Knorsch T, Rogler P, Leick P, Wensing M (2017) High speed shadowgraphy of transparent nozzles as an evaluation tool for in-nozzle cavitation behavior of GDI injectors. In: 28th Conf Liquid Atomization Spray Systems, Valencia, Spain, 06-08 September, pp 1027-103

Mithun MG, Koukouvnis P, Karathanassis IK, Gavaises M (2018) Simulating the effect of in-nozzle cavitation on liquid atomisation using a three-phase model. In: 10th International Symposium on Cavitation, Baltimore, USA, 14-16 Mai, pp 920-924

Mitroglou N, Nouri J, Gavaises M, Arcoumanis C (2006) Spray characteristics of a multi hole injector for direct injection gasoline engines. Int J Engine Res 7:255-270

Moon S, Komada K, Li Z, Wang J, Kimijima T, Arima T, Maeda Y (2015) High-speed X-ray imaging of in-nozzle cavitation and emerging jet flow of multi-hole GDI injector under practical operating conditions. In: 13th Int Conf Liquid Atomization Spray Systems, Tainan, Taiwan, 23-27 August, pp 1-8

Moon S, Komada K, Sato K, Yokohata K, Wada Y, Yasuda N (2015) Ultrafast X-ray study of multi hole GDI injector sprays: effects of nozzle hole length and number on initial spray formation. Exp Therm Fluid Sci 68:68-81

Murai Y, Nakada T, Suzuki T, Yamamoto F (2007) Particle tracking velocimetry applied to estimate the pressure field around a Savonius turbine. Meas Sci Technol 18:2491-2503

Neubert V, Leick P, Stirn R, Dreizler A (2011) Analysis of in-cylinder air motion in a fully optically accessible $2 \mathrm{~V}$ diesel engine by means of conventional and time resolved PIV. In: 9th Int Symp Particle Image Velocimetry, Kobe, Japan, 21-23 July, pp 1-4

Nouri JM, Mitroglou N, Yan Y, Arcoumanis C (2007) Internal flow and cavitation in a multi hole injector for gasoline direct injection engines. SAE Technical Paper 2007-011405. https://doi. org/10.4271/2007-01-1405

Payri F, Bermudez V, Payri R, Salvador VJ (2004) The influence of the cavitation on the internal flow and the spray characteristics in diesel injection nozzles. Fuel 83:419-431

Pennings PC, Bosschers J, Westerweel J, van Terwisga TJ (2015) Dynamics of isolated vortex cavitation. J Fluid Mech 778:288-313

Postrioti L, Bosi M, Cavicchi A, Abuzahra F, Gioia RD, Bonandrini G (2015) Momentum flux measurement on single hole GDI injector under flash boiling conditions. SAE Technical Paper 2015-242480. https://doi.org/10.4271/2015-24-2480

Pröbsting S, Scarano F, Bernardini M, Pirozzoli S (2013) On the estimation of wall pressure coherence using time-resolved tomographic PIV. Exp Fluids 54(7):1-15

Raffel M, Willert CE, Wereley S, Kompenhans J (2007) Particle image velocimetry. Springer, Berlin, Heidelberg

Reid BA, Gavaises M, Mitroglou N, Hargrave GK, Garner CP, Long EJ, McDavid RM (2014) On the formation of string cavitation inside fuel injectors. Exp Fluids 55:1662

Roth H, Gavaises M, Arcoumanis C (2002) Cavitation initiation, its development and link with flow turbulence in diesel injector nozzles. SAE World Congress 2002-01-0214. https://doi. org/10.4271/2002-01-0214

Saha K, Som S, Battistoni M, Li Y, Quan S, Senecal PK (2015) Numerical simulation of internal and near nozzle flow of a gasoline direct fuel injector. J Phys Conf Ser. https://doi.org/10.1088/17426596/656/1/012100

Schmitz I, Ipp W, Leipertz A (2002) Flash boiling effects on the developments of gasoline direct injection sprays. SAE Technical Paper 2002-01-2661. https://doi.org/10.4271/2002-01-2661

Shi J, Arafin MS (2010) CFD investigation of fuel property effect on cavitating flow in generic nozzle geometries. In: 23rd Annu Conf Liquid Atomization Spray Systems, Brno, Czech Republic, 06-09 September, pp 1-10

van Oudheusden BW, Scarano F, Roosenboom EWM, Casimiri EWF, Souverein LJ (2007) Evaluation of integral forces and pressure fields from planar velocimetry data for incompressible and compressible flows. Exp Fluids 43:153-162

Walther J, Schaller JK, Wirth R, Tropea, C. (2000) Investigation of internal flow in transparent diesel injection nozzles using fluorescence particle velocimetry. In: Eighth Int Conf Liquid Atomization Spray Systems, Pasadena, USA, 16-20 July, pp 1-8

Walther J (2002) Quantitative Untersuchungen der Innenströmung in kavitierenden Dieseleinspritzdüsen. Technische Universität Darmstadt, Doktorarbeit

Zigan L, Schmitz I, Wensing M, Leipertz A (2010) Effects of fuel properties on primary breakup and spray formation studied at a gasoline 3 hole nozzle. In: 23rd Annu Conf Liquid Atomization Spray Systems, Brno, Czech Republic, 06-09 September, pp 1-8

Zheng Y (2013) Simulations and experiments of fuel injection, mixing and combustion in DI gasoline engines. Ph.D. Thesis, Wayne State University

Publisher's Note Springer Nature remains neutral with regard to jurisdictional claims in published maps and institutional affiliations. 


\section{Affiliations}

\section{Dmitrii Mamaikin $^{1}$ (D) Tobias Knorsch $^{2} \cdot$ Philipp Rogler $^{2} \cdot$ Michael Wensing $^{1}$}

\author{
Institute of Engineering Thermodynamics, FAU Erlangen- \\ Nuremberg, Am Weichselgarten 8, 91058 Erlangen, \\ Germany
}

2 Robert Bosch GmbH, Powertrain Solutions,

Robert-Bosch-Strasse 2, 71701 Schwieberdingen, Germany 